\title{
Analysis of the role of branching angle in the dynamic rupture process on a 3-D branching fault system
}

\author{
JingXing Fang, Feng Qian, and HaiMing Zhang* \\ School of Earth and Space Sciences, Peking University, Beijing 100871, China
}

\section{Key Points:}

- Numerical simulations of rupture propagation on 3-D faults using BIEM and friction law dependent on normal stress

- The propensity of fault planes of different angles to rupture can be analyzed explicitly by Mohr-Coulomb diagram

- The upper and lower branch of a branching fault effect each other in different patterns

Citation: Fang, J. X., Qian, F., and Zhang, H. M. (2020). Analysis of the role of branching angle in the dynamic rupture process on a 3-D branching fault system. Earth Planet. Phys., 4(5), 523-531. http://doi.org/10.26464/epp2020043

\begin{abstract}
The fault branching phenomenon, which may heavily influence the patterns of rupture propagation in fault systems, is one of the geometric complexities of fault systems that is widely observed in nature. In this study, we investigate the effect of the branching angle on the rupture inclination and the interaction between branch planes in two-fork branching fault systems by numerical simulation and theoretical analysis based on Mohr's circle. A friction law dependent on normal stress is used, and special attention is paid to studying how ruptures on the upper and lower branch planes affect the stress and rupture on each other separately. The results show that the two branch planes affect each other in different patterns and that the intensity of the effect changes with the branching angle. The rupture of the lower branch plane has a negative effect on the rupture of the upper branch plane in the case of a small branching angle but has almost no negative effect in the case of a large branching angle. The rupture of the upper branch plane, however, suppresses the rupture of the lower branch plane regardless of whether the branching angle is large or small.
\end{abstract}

Keywords: branching faults; Mohr-Coulomb diagram; boundary integral equation method; earthquake source dynamics; rupture selectivity

\section{Introduction}

Branching faults are widely observed in nature, and many natural earthquakes involve them, such as the $2008 M_{\mathrm{s}} 8.0$ Wenchuan earthquake (Hao KX et al., 2009), the $1979 M_{\mathrm{w}} 6.4$ Imperial Valley earthquake (Niazi, 1986), the $1992 M_{\mathrm{w}} 7.3$ Landers earthquake (Sieh et al., 1993) and the $2002 M_{s} 7.9$ Denali earthquake (Eberhart-Phillips et al., 2003). Because fault branching is one of the complexities responsible for the complex patterns of earthquake source ruptures, it is important to study the dynamic rupture behavior of branching faults for earthquake hazard assessment and disaster prevention.

Since the 1990s, seismologists have made it technically possible to consider fault branching in the research on earthquake source dynamics. Because the boundary integral equation method (BIEM) includes the feature of reducing the dimensions of the considered problem by one, the BIEM is advantageous in dealing with complex fault geometry. Perhaps the simplest case is a static antiplane crack on branching faults, which was first considered by Tada and Yamashita (1997). Kame and Yamashita (1999) then sim-

Correspondence to: H. M. Zhang, zhanghm@pku.edu.cn

Received 12 FEB 2020; Accepted 21 APR 2020.

Accepted article online 12 JUN 2020.

(C) 2020 by Earth and Planetary Physics. ulated the spontaneous bifurcation of a crack tip. However, neither study specifically focused on the geometry of branching faults. Poliakov et al. (2002) theoretically analyzed the stress field around a dynamic crack tip and predicted how the prestress state would affect the most favored direction for dynamic branching of a rupture. Kame et al. (2003) conducted simulations of dynamic rupture processes on branching faults and studied the effects of the prestress state, branching angle, and rupture velocity on the propensity to rupture. Bhat et al. (2007) studied the effect of branch length and predicted the interaction between a rupture on the main fault and on the branch plane. All the above studies used 2-D fault models. Aochi et al. (2000a) constructed a nonsingular BIEM in a 3-D elastic medium, which was used to study the effect of branching angles on the interactions between planes of branching faults (Aochi et al., 2000b) and the effect of normal stress on the rupture selectivity on branching faults (Aochi et al., 2002). Recently, Ando and Kaneko (2018) studied the effect of complex 3-D fault geometry on rupture propagation and termination during the $2016 M_{\mathrm{w}} 7.9$ Kaikoura earthquake, which occurred on branching faults.

Other simulation methods, such as the finite element method (FEM), have also been used to study the rupture dynamics of branching faults. Oglesby et al. (2003) simulated the $1999 M_{\mathrm{w}} 7.1$ Hector Mine earthquake on a complex fault system with a branch- 
ing fault to the north. DeDontney et al. (2012) investigated the earthquake rupture behavior on branching faults in an elastic-plastic medium. Xu DD et al. (2016) computed the dynamic ruptures along branching faults in the Longmen Mountain fault and analyzed the effects of the prestress state and fault geometry on rupture path selection. A limitation of the FEM is that different artificial treatments on the branching junctions in branching faults may result in different simulation outcomes (DeDontney et al., 2012).

Our study aimed to determine the effect of branching angles on the rupture propagation on branching faults and provide a physical explanation. This topic has been studied previously. Kame et al. (2003) pointed out the interactions between two branch planes, but they did not clarify the mechanism of the interactions. Aochi et al. (2000b) and Dong S and Zhang HM (2019) studied the stress interaction between the two branch planes, but the friction law they used was independent of normal stress, and they did not separate the stress effect generated by each part of the fault system. In this research, we simulated the dynamic propagation of a rupture on a 3-D two-fork branching fault model more realistically by using the BIEM with the slip-weakening Coulomb friction law (Kame et al., 2003), which is dependent on normal stress. First, the inclination of different angles of branch planes to rupture was analyzed explicitly by using a Mohr-Coulomb diagram. The stress accumulations produced by the slip of one of the branch planes on the other were then separated to analyze how the interactions between the two branch planes were affected by the branching angle.

\section{Method and Mode}

We used the BIEM to simulate dynamic rupture propagation. By considering an unstructured mesh of a branching fault system containing $N_{x}$ triangular elements, the discretized boundary integral equation can be written as (Qian F et al., 2019)

$$
T_{m p}=T_{m}^{0}+\sum_{n=0}^{N_{x}} \sum_{q=1}^{p} V_{n q} K_{m p / n q}
$$

where $T_{m p}$ is the stress of element $m$ at time $p, T_{m}^{0}$ is the initial stress of element $m, K_{m p / n q}$ is the stress kernel based on the triangular elements (Feng X and Zhang HM, 2017), which represents the stress increment of element $m$ at time $p$ generated by the unit slip of element $n$ at time $q$, and $v_{n q}$ is the slip rate of element $n$ at time $q$. Equation (1) implies that the stress change of an element is influenced by the previous slip history of all elements, and the contribution of the slip of each element can clearly be calculated. Therefore, by using the BIEM, we can explicitly analyze the interaction between any two segments of a fault system.

As is usually adopted in the simulation of spontaneous dynamic ruptures, the slip-weakening friction law (Ida, 1972) was introduced in this study. For any given element on the fault system, it begins to rupture as soon as the shear stress on which exceeds the peak strength (yield stress) $\tau_{p}$, and the shear stress decreases linearly with the increase in its slip distance until it reaches the residual stress level $\tau_{r}$, as shown in Figure 1. Variable $D_{c}$ is the slipweakening distance related to the rock property and is set at 0.57 $\mathrm{m}$ in this study. We introduced the Coulomb criteria into the slip- weakening friction law (Kame et al., 2003), which defines $\tau_{p}$ and $\tau_{r}$ of an element proportional to its normal stress, and the scale factors were the static and dynamic friction coefficients, $\mu_{s}$ and $\mu_{d}$, respectively. By combining the slip-weakening friction law and the BIEM, we could determine the slip rate of each element at each time step, based on which the corresponding slip and stress could also be obtained.

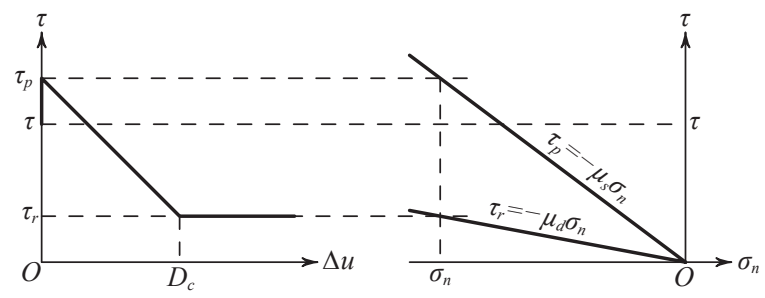

Figure 1. Slip-weakening Coulomb friction law (Kame et al., 2003), where $\sigma_{n}$ is the normal stress, $D_{c}$ is the slip-weakening distance, and $\mu_{s}$ and $\mu_{d}$ are static and dynamic friction coefficients, respectively. The peak strength $\left(\tau_{p}\right)$, the residual stress $\left(\tau_{r}\right)$, and the shear stress $(\tau)$ at any amount of slip $(\Delta u)$ is proportional to the normal compressive stress $\left(\sigma_{n}\right)$.

In this research, we considered a branching fault system in a 3-D unbounded homogeneous isotropic elastic medium, as shown in Figure 2a. Previous studies have shown that when a fault system is located at a depth of more than $1 \mathrm{~km}$ below the surface, the influence from the surface is very small and can be neglected (Zhang HM and Chen XF, 2006). The entire branching fault system consisted of three fault planes: a $30 \mathrm{~km} \times 10 \mathrm{~km}$ primary plane $a$ and two $15 \mathrm{~km} \times 10 \mathrm{~km}$ branch planes $b$ and $c$. We used the Cartesian coordinate system and took point $A$ as the origin of the coordinate, with the $x$ and $y$ axes being parallel to $O A$ and $O O^{\prime}$, respectively. The angle between planes $a$ and $b$ and that between planes $a$ and $c$ are denoted as $a_{1}$ and $a_{2}$, both of which are counted counterclockwise. The fault model was discretized by unstructured triangular elements. Elements I, II, and III lie on the midline of the fault system and are next to the intersection, as shown in Figure $2 \mathrm{~b}$ and $2 \mathrm{c}$. The minimum of the inscribed circle radiuses of all the triangular elements was $249 \mathrm{~m}$, and the time step $\Delta t$ of our simulation was $0.044 \mathrm{~s}$. The density of the medium was 3,000 $\mathrm{kg} / \mathrm{m}^{3}$, and the P-wave and S-wave velocities in the medium were $5.6 \mathrm{~km} / \mathrm{s}$ and $3.2 \mathrm{~km} / \mathrm{s}$, respectively.

The entire fault system was loaded with a uniform initial stress field. It was assumed that the shear and normal stresses on planes $a, b$, and $c$ had no $y$ component, and neither did the slip on each plane. As is shown in Figure $2 a$, a round nucleation zone with a radius of $2.5 \mathrm{~km}$ was located at the center of plane $a$, in which the shear stress was slightly greater than $\tau_{p}$. The rupture began from the nucleation zone and propagated outward spontaneously. Given the initial stress components $\tau_{x x}^{0}, \tau_{z z}^{0}$, and $\tau_{x z}^{0}$, the normal and shear stresses on each plane could be determined, and the spatiotemporal history of the rupture process in order of time could then be calculated by using the BIEM and the friction law. Throughout this study, $\tau_{x x}^{0}=\tau_{z z}^{0}=-100 \mathrm{MPa}$, and $\tau_{x z}^{0}=50 \mathrm{MPa}$. The initial shear stress in the nucleation zone was $1.2 \tau_{p}$ to generate an 
(a)

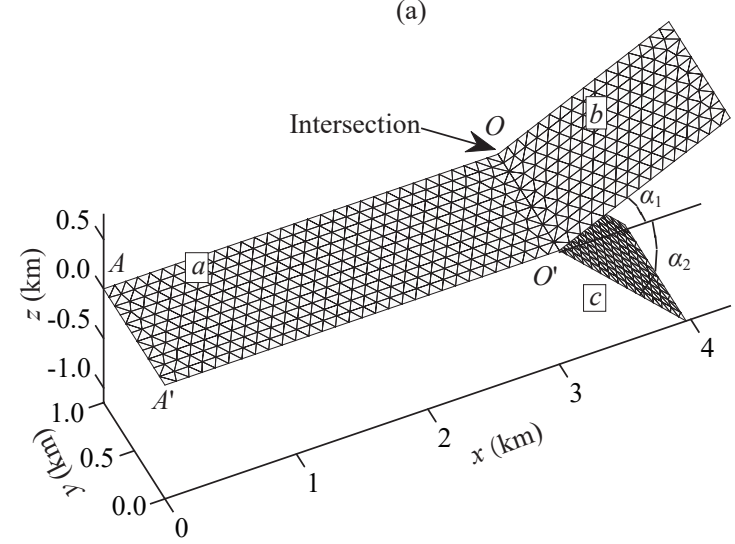

(b)
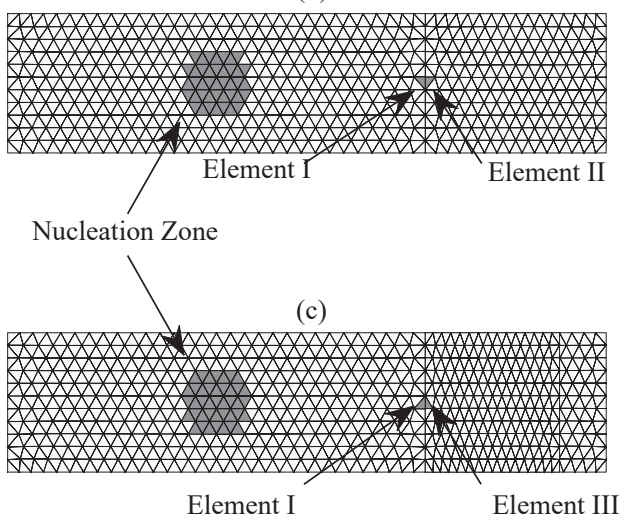

Figure 2. Sketch of the fault model used in this study. (a) The entire fault system, which consists of three subfaults, $a, b$, and $c$. The angle measured from plane $a$ to plane $b$ is $a_{1}$, and the angle measured from plane $a$ to plane $c$ is $a_{2}$. Both $a_{1}$ and $a_{2}$ are counted counterclockwise. The fault system is divided into unstructured triangular elements. The positions of the nucleation zone and the intersection are denoted by arrows. (b) Viewed from the top of the model, elements I and II are the gray triangles denoted by arrows, respectively. (c) Viewed from the bottom of the model, elements I and III are the gray triangles denoted by arrows, respectively.

initial crack. Because we were primarily focusing on the effect of $a_{1}, a_{2}$ on the rupture patterns of planes $b$ and $c$ and the interaction between them in this study, all the values of geometric and physical quantities except $a_{1}$ and $a_{2}$ were fixed in our simulations.

\section{Numerical Results}

In our simulations, $a_{1}$ was always greater than $a_{2}$, which always placed plane $b$ above plane $c$. Therefore, the included angle of planes $b$ and $c, \Delta a=a_{1}-a_{2}$, were always positive. The rupture processes on two series of faults were computed: series 1 , in which $a_{1}=10^{\circ}$ remained constant, and $a_{2}=-10^{\circ},-20^{\circ},-25^{\circ},-30^{\circ}$, $-40^{\circ}$, and series 2 , in which $a_{2}=-25^{\circ}$ remained constant, and $a_{1}=15^{\circ}, 10^{\circ}, 5^{\circ},-5^{\circ},-15^{\circ}$. Analysis of the numerical results will be helpful in understanding the effect of branching angles on how a rupture on the upper or lower branch planes separately influences rupture propagation on the other branch plane.

Figures 3 and 4 are space-time diagrams of the slip rate on different faults in series 1 and 2 . For comparison, we also computed the rupture processes on two folding faults, one of which consisted of only planes $a$ and $b$, with $a_{1}=10^{\circ}$ (Figure 3a), and the other of which consisted of only planes $a$ and $c$, with $a_{2}=-25^{\circ}$ (Figure 4a), to show the rupture front patterns on one branch plane without the influence of the other branch plane. Because the rupture patterns on the primary plane $a$ were barely affected by the rupture on plane $b$ or $c$ with different $a_{1}$ and $a_{2}$, results on plane $a$ are only shown in Figures $3 a$ (distance of 0 to $15 \mathrm{~km}$ ) and $4 \mathrm{a}$ (distance of 0 to $15 \mathrm{~km}$ ).

\subsection{Results of Series 1}

Figure 3a shows that in a folding fault system where plane $c$ does not exist, the rupture propagates through the folding line and reaches the farthest edge of plane $c$ at around $t=299 \Delta t$. For a branching fault system (Figures $3 b-3 f$ ), on the other hand, the rupture on plane $c$ is dominant in the cases of $a_{2}=-10^{\circ},-20^{\circ}$, and $-25^{\circ}$. In the case of $a_{2}=-30^{\circ}$ (Figure $3 \mathrm{e}$ ), the rupture propagates almost equally on both planes $b$ and $c$, and when $a_{2}=-40^{\circ}$ (Figure $3 f$ ), the rupture on plane $b$ becomes dominant. In all five cases of different $a_{2}$ values, the rupture on plane $b$ is suppressed in comparison with the folding fault case (Figure $3 a$ ), and the suppression becomes stronger as the absolute value of $a_{2}$ becomes smaller, i.e., $\Delta a$ becomes smaller. As shown in Figure $5 a$, the rupture propagation velocities on plane $b$ in different cases can be compared in the space-time diagram of the rupture front. The corresponding result on plane $b$ in the folding fault model is also shown for reference. Note that the rupture propagation velocity on plane $b$ when $a_{2}=-40^{\circ}$ is almost the same as that of the folding fault. In other cases, this velocity becomes smaller with a smaller $\Delta a$.

In Table 1, we list the times when elements I, II, and III begin to crack in different cases of series 1 , which indicate the times when the rupture reaches the intersection and begins on planes $b$ and $c$, respectively. In cases $a_{2}=-10^{\circ},-20^{\circ}$, and $-25^{\circ}$, plane $c$ cracks before the rupture front on plane $a$ reaches the intersection, whereas plane $b$ cracks after the rupture on plane $a$ reaches the intersection. In cases $a_{2}=-30^{\circ}$ and $-40^{\circ}$, both planes $b$ and $c$ crack after the rupture on plane $a$ reaches the intersection. Generally, the larger the branching angle is, the sooner plane $b$ cracks, and the later plane $c$ cracks.

\subsection{Results of Series 2}

Figure $4 a$ shows that the rupture can propagate to and throughout plane $c$ without an effect on plane $b$. The rupture on plane $c$ dominates in cases with $a_{1}=15^{\circ}$ and $10^{\circ}$, whereas the rupture on plane $b$ dominates in cases with $a_{1}=-5^{\circ}$ and $-15^{\circ}$. When $a_{1}=5^{\circ}$, the rupture propagates almost equally on planes $b$ and $c$. The slip rate on plane $c$ decreases slightly from $a_{1}=15^{\circ}$ to $5^{\circ}$ and violently when $a_{1}=-5^{\circ}$ and $-15^{\circ}$. In Figure $5 \mathrm{~b}$, the rupture front propagates at almost the same velocity on plane $c$ in cases with $a_{1}=15^{\circ}, 10^{\circ}$, and $5^{\circ}$ and at a slightly slower velocity when $a_{1}=-5^{\circ}$. In contrast, in the case of $a_{1}=-15^{\circ}$, the crack on plane $c$ obviously begins late and stops quickly. 

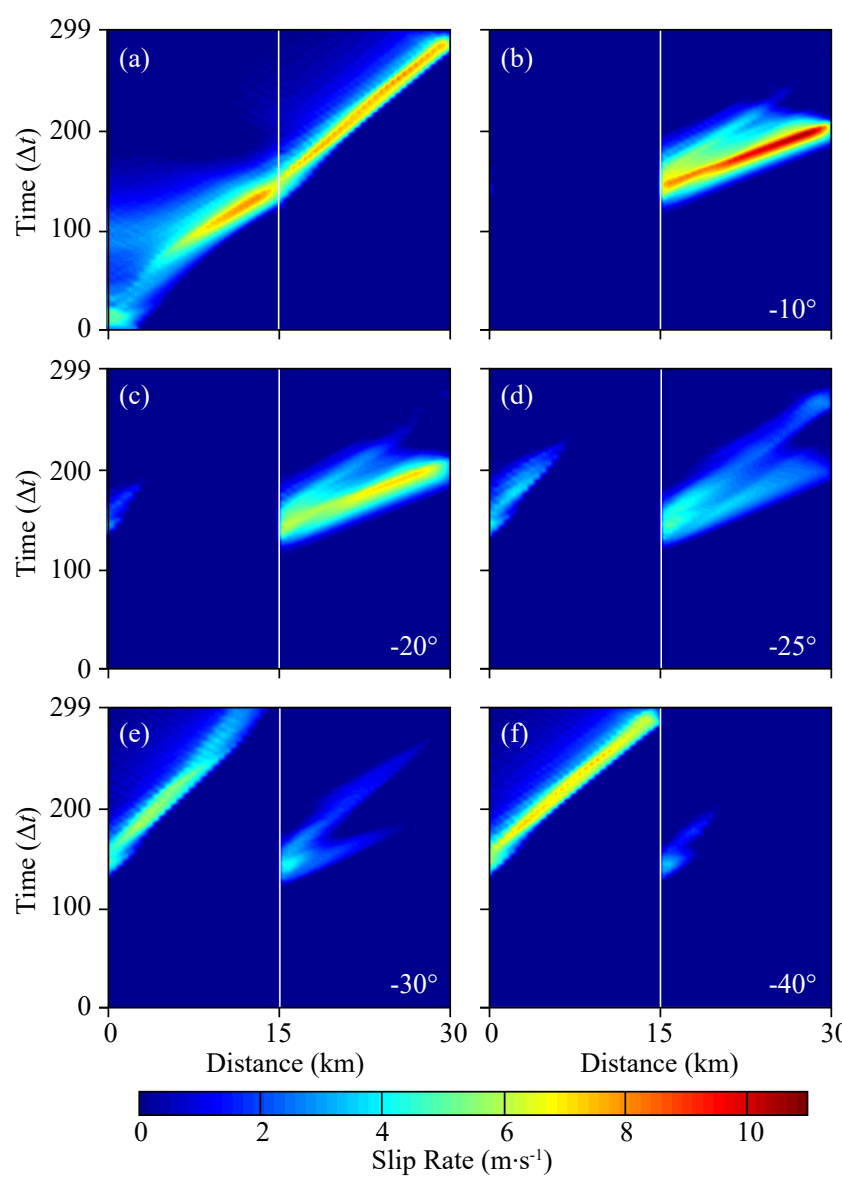

Figure 3. Space-time diagrams of the slip rate on the midline $(y=5$ $\mathrm{km}$ ) along the faults of series 1. (a) Results for a folding fault consisting of planes $a$ and $b$ only (without plane $c$ ). The distance 0 to $15 \mathrm{~km}$ corresponds to the part of $x=15$ to $30 \mathrm{~km}$ on plane $a$ (from the nucleation zone to the intersection), and the distance 15 to $30 \mathrm{~km}$ corresponds to plane $b$. (b-f) Results for series 1 with $a_{2}=-10^{\circ},-20^{\circ},-25^{\circ},-30^{\circ}$, and $-40^{\circ}$, respectively. The distance 0 to $15 \mathrm{~km}$ corresponds to plane $b$ ), and the distance 15 to $30 \mathrm{~km}$ corresponds to plane $c$.

Table 2 shows the times when the rupture reaches the intersection and begins on planes $b$ and $c$. In cases with $a_{1}=15^{\circ}, 10^{\circ}$, and $5^{\circ}$, plane $c$ cracks before the rupture on plane $a$ reaches the intersection, after which plane $b$ cracks. When $a_{1}=-5^{\circ}$, planes $c$ and $b$ both crack before the rupture on plane $a$ arrives at the intersection, and plane $c$ cracks sooner than plane $b$. Different from this scenario, when $a_{1}=-15^{\circ}$, plane $b$ cracks first, and then the rupture on plane $a$ arrives at the intersection and plane $c$ cracks at almost the same time.

\section{Physical Explanations}

\subsection{Effect of Branching Angles on the Chronological Order of Cracks}

As shown in Tables 1 and 2, the chronological order at which elements I, II, and III begin to crack varies with the values of $a_{1}$ and $a_{2}$, which can be illustrated by the Mohr-Coulomb diagram shown in Figure 6.
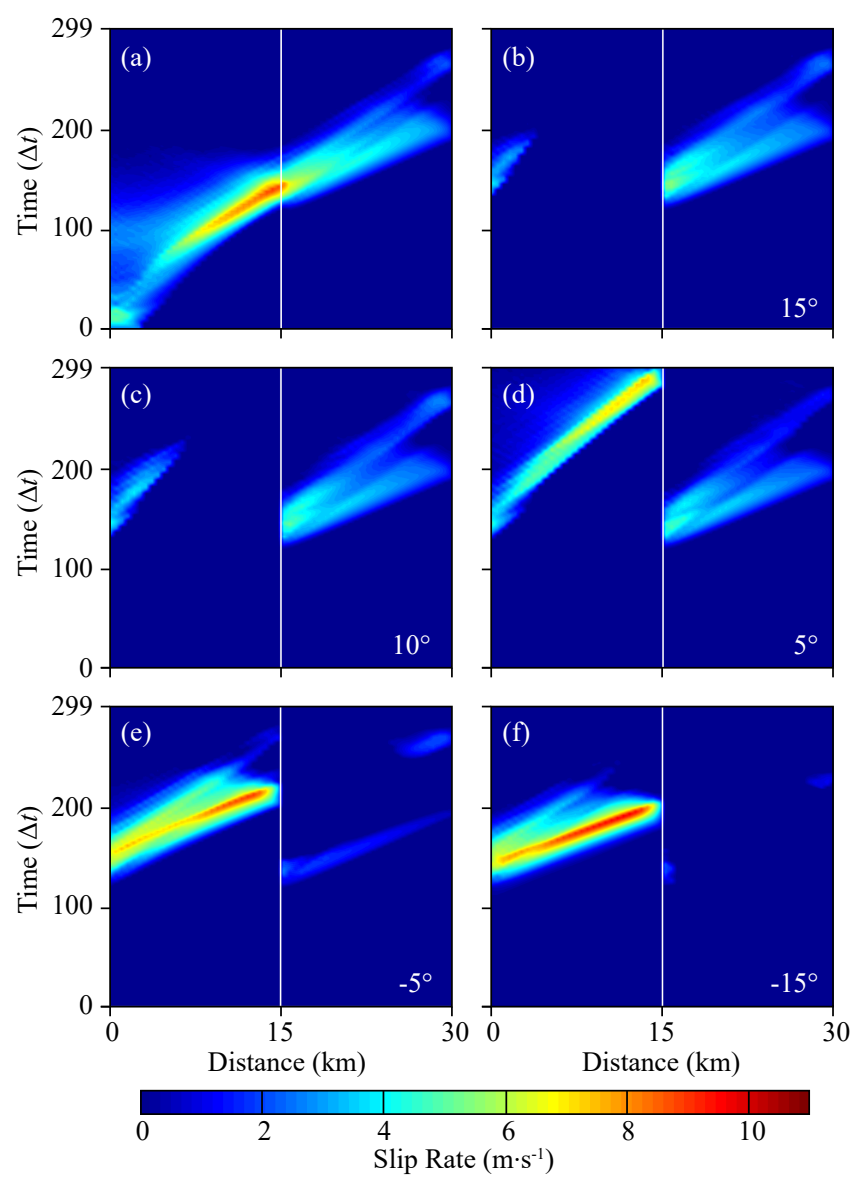

Figure 4. Same as Figure 3 except for series 2 here. (a) Results for a folding fault consisting of planes $a$ and $c$ only (without plane $b$ ). The distance 0 to $15 \mathrm{~km}$ corresponds to the part of $x=15$ to $30 \mathrm{~km}$ on plane $a$ (from the nucleation zone to the intersection), and the distance 15 to $30 \mathrm{~km}$ corresponds to plane $c$. (b-f) Results for series 2 with $a_{1}=15^{\circ}, 10^{\circ}, 5^{\circ},-5^{\circ}$, and $-15^{\circ}$, respectively. The distance 0 to 15 $\mathrm{km}$ corresponds to plane $b$, and the distance 15 to $30 \mathrm{~km}$ corresponds to plane $c$.

Given the initial stress field, we can draw a Mohr's circle, on which any plane with a certain direction in this stress field can be donated as a point on the circle. We introduce the slip-weakening Coulomb friction law into the Mohr's circle to evaluate the inclination of cracking for different planes. A point close to the peak strength line marked by $\tau_{p}$ in Figure 6 on the Mohr's circle corresponds to a plane that cracks easily. A plane is not permitted to crack if its initial shear stress is less than the residual stress level marked by $\tau_{r}$ in Figure 6 (Aochi et al., 2000a). The direction of the plane that cracks the easiest can be defined as the optimal plane, as shown below:

$$
\Phi_{\mathrm{opt}}=\frac{\pi}{4}-\frac{1}{2} \tan ^{-1} \mu_{s^{\prime}}
$$

where $\Phi_{\mathrm{opt}}$ is measured from the maximum principal stress direction. We can then estimate the preference for rupture propagation by measuring the angle between a particular plane and the optimal plane. In this study, the angle measured from plane $a$ to the optimal plane was $-15.5^{\circ}$ (counted counterclockwise). 

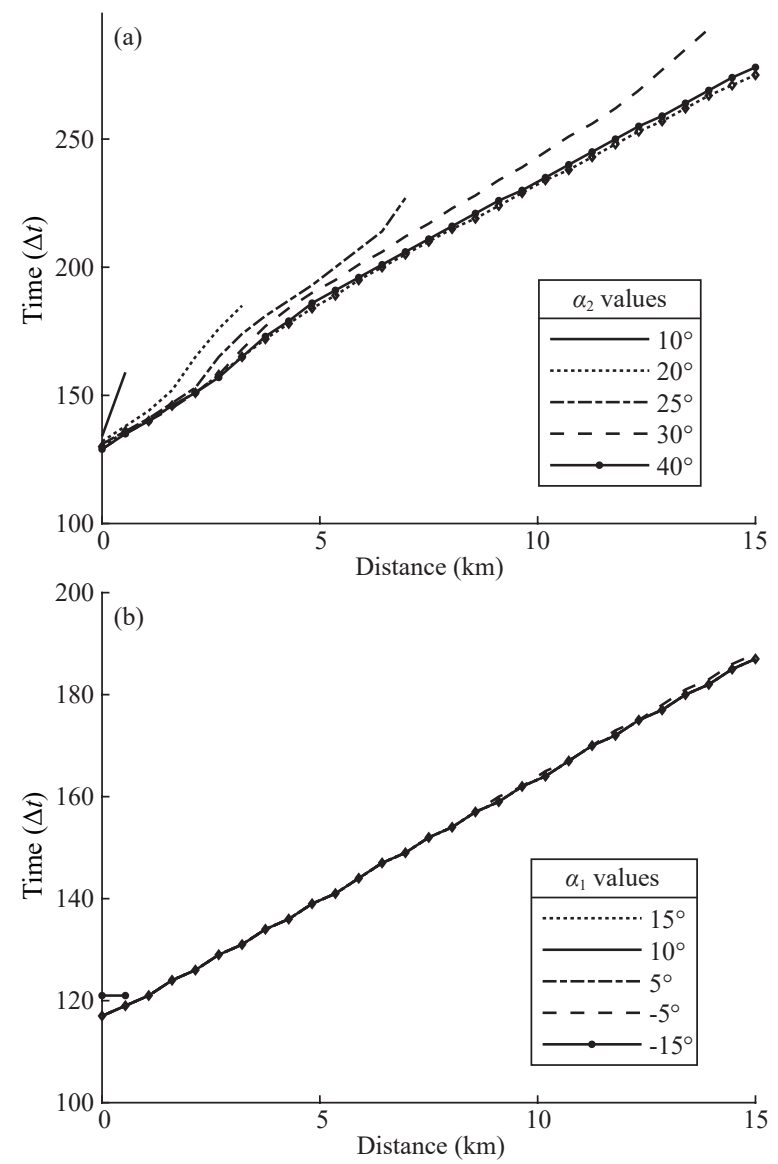

Figure 5. Time-distance diagrams of rupture fronts (a) on plane $b$ in series 1 and (b) on plane $c$ in series 2 . The gray dotted lines in (a) and (b) illustrate the rupture fronts on folding planes $b$ and $c$ with $a_{1}=10^{\circ}$ and $a_{2}=-25^{\circ}$, respectively.

Table 1. Times at which elements I, II, and III begin to crack $\left(t_{c}\right)$ for the simulations in series 1 .

\begin{tabular}{cccc}
\hline & \multicolumn{3}{c}{$t_{c} / \Delta t$} \\
\cline { 2 - 4 }$a_{2}$ & Element I & Element II & Element III \\
\hline$-10^{\circ}$ & 120 & 134 & 112 \\
$-20^{\circ}$ & 119 & 132 & 110 \\
$-25^{\circ}$ & 120 & 131 & 117 \\
$-30^{\circ}$ & 120 & 129 & 122 \\
$-40^{\circ}$ & 120 & 129 & 127 \\
\hline
\end{tabular}

In series 1 , because plane $a$ is closer to the optimal plane than is plane $b$, the crack cannot begin on plane $b$ unless the rupture has reached the intersection. Plane $c$, with $a_{2}=-10^{\circ},-20^{\circ}$, and $-25^{\circ}$, is closer to the optimal plane than is plane $a$. Thus, element III cracks before element I does. In series 2, element III cracks before element I does when $a_{1} \geq-5^{\circ}$ because plane $c$ is closer to the optimal plane than is plane $a$.

The Mohr-Coulomb diagram can explain only the preference for rupture propagation before either of the two branch planes cracks because a branch plane will produce a stress effect on the
Table 2. Times at which elements I, II, and III begin to crack $\left(t_{c}\right)$ for the simulations in series 2 .

\begin{tabular}{cccc}
\hline & \multicolumn{3}{c}{$t_{c} / \Delta t$} \\
\cline { 2 - 4 }$a_{1}$ & Element I & Element II & Element III \\
\hline $15^{\circ}$ & 120 & 132 & 117 \\
$10^{\circ}$ & 119 & 131 & 117 \\
$5^{\circ}$ & 120 & 128 & 117 \\
$-5^{\circ}$ & 120 & 119 & 117 \\
$-15^{\circ}$ & 120 & 107 & 121 \\
\hline
\end{tabular}

other once it cracks. Take $a_{1}=-15^{\circ}$ and $a_{2}=-25^{\circ}$ as an example. Plane $c$ is supposed to crack before the rupture arrives at the intersection because it is closer to the optimal plane than is plane $a$. In fact, however, it cracks after the rupture reaches the intersection because plane $b$ cracks first and thereafter affects the rupture on plane $c$.

\subsection{Effect of the Lower Branch on the Upper One}

Because element II is the first element on plane $b$ to crack, the stress change on plane $b$ indicates its potential for rupture growth. In series 1, we investigated the normal and shear stress change on element II produced by the rupture on planes $a$ and $c$, denoted as $T_{\text {element II, } p}^{a}$ and $T_{\text {element II, } p}^{c}$ separately, by changing the sum in Equation (1) as follows

$$
\begin{aligned}
& T_{\text {element II, } p}^{a}=T_{\text {element II, } 0}^{a}+\sum_{n=n_{1}}^{n_{2}} \sum_{q=1}^{p} V_{n q} K_{m p / n q} \\
& T_{\text {element II, } p}^{c}=T_{\text {element II, } 0}^{c}+\sum_{n=n_{3}}^{n_{4}} \sum_{q=1}^{p} V_{n q} K_{m p / n q \prime}
\end{aligned}
$$

where elements numbered from $n_{1}$ to $n_{2}$ are all the elements on plane $a$ and those numbered from $n_{3}$ to $n_{4}$ are all the elements on plane $c$. The slip rate on plane $a$ is almost independent of planes $b$ and $c$, so according to Equation (3), its effect on the stress field of plane $b$ barely changes with $a_{2}$. We show this effect alone in Figure $7 \mathrm{a}$ to indicate that the rupture on plane $a$ promotes the cracking of plane $b$. The effects of plane $c$ alone on element II with different $a_{2}$ are shown in Figure $7 \mathrm{~b}-7 \mathrm{f}$.

In the case of $a_{2}=-10^{\circ}$ (Figure $7 \mathrm{~b}$ ), the shear stress decreases rapidly while the normal stress decreases slowly on element II, quickly moving the stress on element II away from the peak strength line on the Mohr-Coulomb diagram. The stress variation on element II produced by plane $c$ is almost opposite that produced by plane $a$. Thus, if plane $c$ cracks prior to plane $b$, it rapidly produces a negative effect on the rupture of plane $b$, which is in accordance with the fact that plane $b$ cracks both very late and very weakly when $\left(a_{1}, a_{2}\right)=\left(10^{\circ},-10^{\circ}\right)$. When $a_{2}=-20^{\circ}$ and $-25^{\circ}$ (Figure $7 \mathrm{c}$ and $7 \mathrm{~d}$ ), the normal stress decreases more quickly and the shear stress decreases more slowly on element II. The stress on element II still moves away from the peak strength line, but at a slower speed. When $a_{2}=-30^{\circ}$ (Figure 7e), plane $c$ cracks before plane $b$, but the effect of the rupture on plane $c$ slowly moves the stress on element II away from the peak strength line so that the rupture can begin on plane $b$ shortly after plane $c$ cracks and propagates throughout plane $b$. Finally, when $a_{2}=-40^{\circ}$, the rup- 

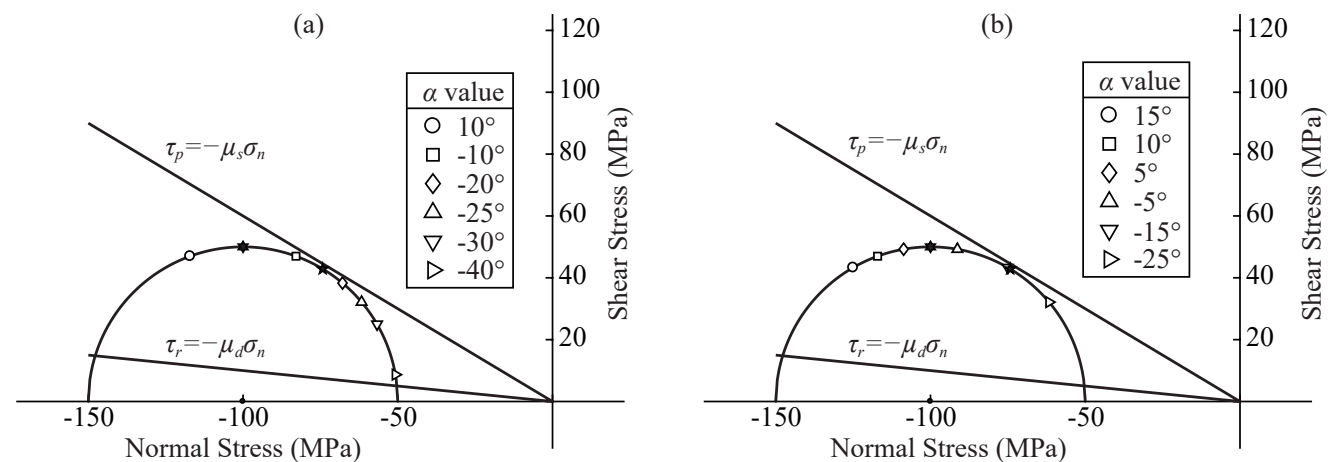

Figure 6. Mohr-Coulomb diagram for planes with different dips in (a) series 1 and (b) series 2. $a$ is the angle between plane $a$ and a branch plane $b$ or $c$. The black filled hexagrams represent plane $a$, and the black filled pentagrams represent the optimal plane. Lines $\tau_{p}=-\mu_{s} \times \sigma_{n}$ and $\tau_{r}=-\mu_{d} \times \sigma_{n}$ are the peak strength and residual stress line, with the static and dynamic friction coefficients $\mu_{s}=0.6$ and $\mu_{d}=0.1$, respectively.
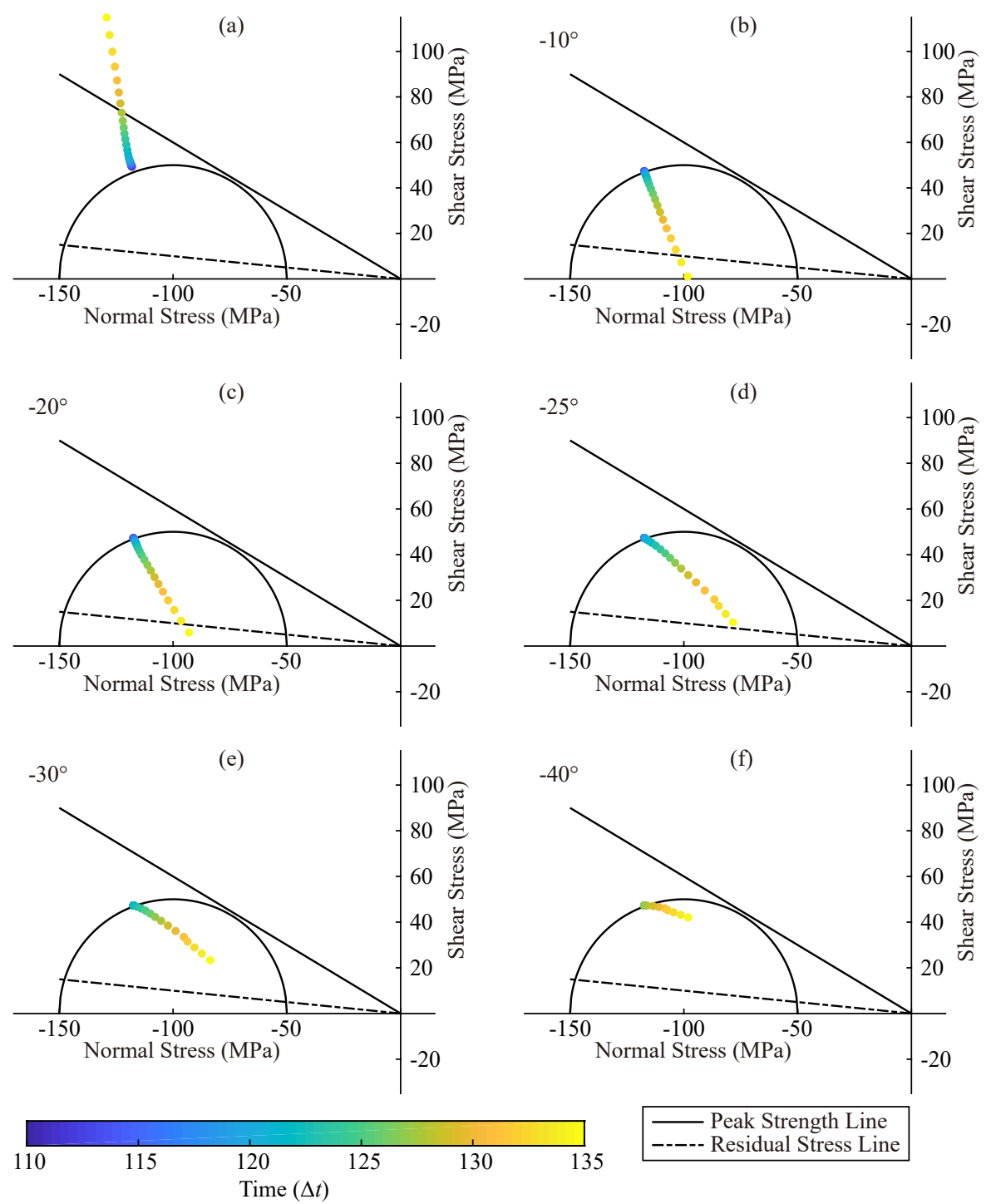

Figure 7. Normal and shear stress on element II at time steps 110 to 135 if we consider only the influence of the rupture of (a) plane $a$ in the case $\left(a_{1}, a_{2}\right)=\left(10^{\circ},-10^{\circ}\right)$, and $(\mathrm{b}-\mathrm{f})$ plane $c$ in series 1 with different $a_{2}\left(a_{2}=-10^{\circ},-20^{\circ},-25^{\circ},-30^{\circ}\right.$, and $\left.-40^{\circ}\right)$. The ordinate and abscissa of a dot represent the shear stress and normal stress on element II, respectively, and the color represents the time of the dot. 

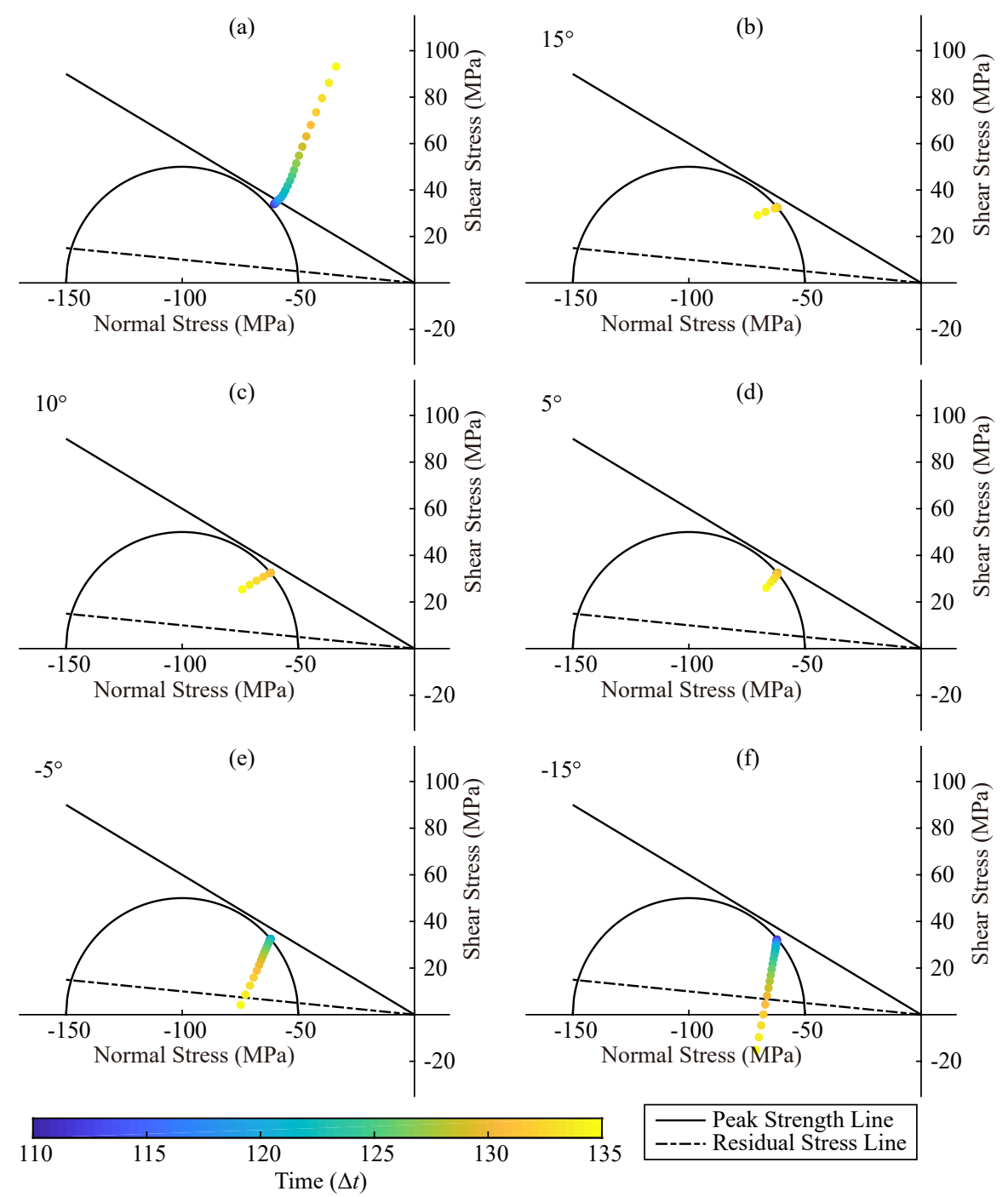

Figure 8. Normal and shear stress on element III at time steps 110 to 135 if we consider only the influence of the rupture of (a) plane $a$ in the case $\left(a_{1}, a_{2}\right)=\left(10^{\circ},-25^{\circ}\right)$, and $(\mathrm{b}-\mathrm{f})$ plane $b$ in series 2 with different $a_{1}\left(a_{1}=15^{\circ}, 10^{\circ}, 5^{\circ},-5^{\circ}\right.$, and $\left.-15^{\circ}\right)$. The ordinate and abscissa of a dot represent the shear stress and normal stress on element III, respectively, and the color represents the time of the dot.

ture of plane $c$ is weak, and the trend of its effect on element II (Figure $7 f$ indicates that the rupture on plane $b$ is scarcely impeded.

Generally, the rupture on plane $c$ (i.e., the lower branch) reduces both the normal stress and the shear stress on element II. As $\Delta a=a_{1}-a_{2}$ increases, the shear stress decreases more slowly and the normal stress decreases more quickly, which lessens the suppression on the rupture of plane $b$.

\subsection{Effect of the Upper Branch on the Lower One}

In series 2 , we calculated the stress variation on element III generated by planes $a$ and $b$, as shown in Figure 8 . Because the rupture on plane $a$ is barely affected by the ruptures on planes $b$ and $c$, its effect on the stress on element III changes little in different cases in series 2. Figure 8a shows that the rupture on plane $a$ causes the stress on element III to approach the peak strength line quickly, which promotes its cracking.

When $a_{1}=15^{\circ}, 10^{\circ}$, and $5^{\circ}$ (Figure $8 \mathrm{~b}-8 \mathrm{~d}$ ), the rupture on plane $b$ moves the stress on element III away from the peak strength line, but this effect is very weak because of the suppressed rupture on plane $b$. To investigate specifically how the rupture on plane $b$ affects the stress on element III without the influence of plane $a$ resulting from different $a_{1}$ in these three cases, we assumed that the direction of plane $a$ equaled that of plane $b$ and that plane $c$ did not produce any stress effect on plane $b$ so that the rupture on plane $a$ would propagate to plane $b$ unimpeded. We then calculated the effect of plane $b$ on the stress on element $c$, as shown in Figure 9. The only difference between Figure 9 and Figure $8 b-8 d$ is that the ruptures on plane $b$ in Figure 9 are stronger than those in Figure 8b-8f.

Figure $8 \mathrm{e}-8 \mathrm{f}$ and Figure 9 show that the rupture on plane $b$ increases the normal stress and decreases the shear stress on element III. When the value of $\Delta a$ is large, the normal stress increases more rapidly and the shear stress decreases more slowly, whereas the normal stress increases more slowly and the shear stress decreases more rapidly when $\Delta a$ becomes smaller. Within the range of $\Delta a$ investigated in this study, the stress on element 


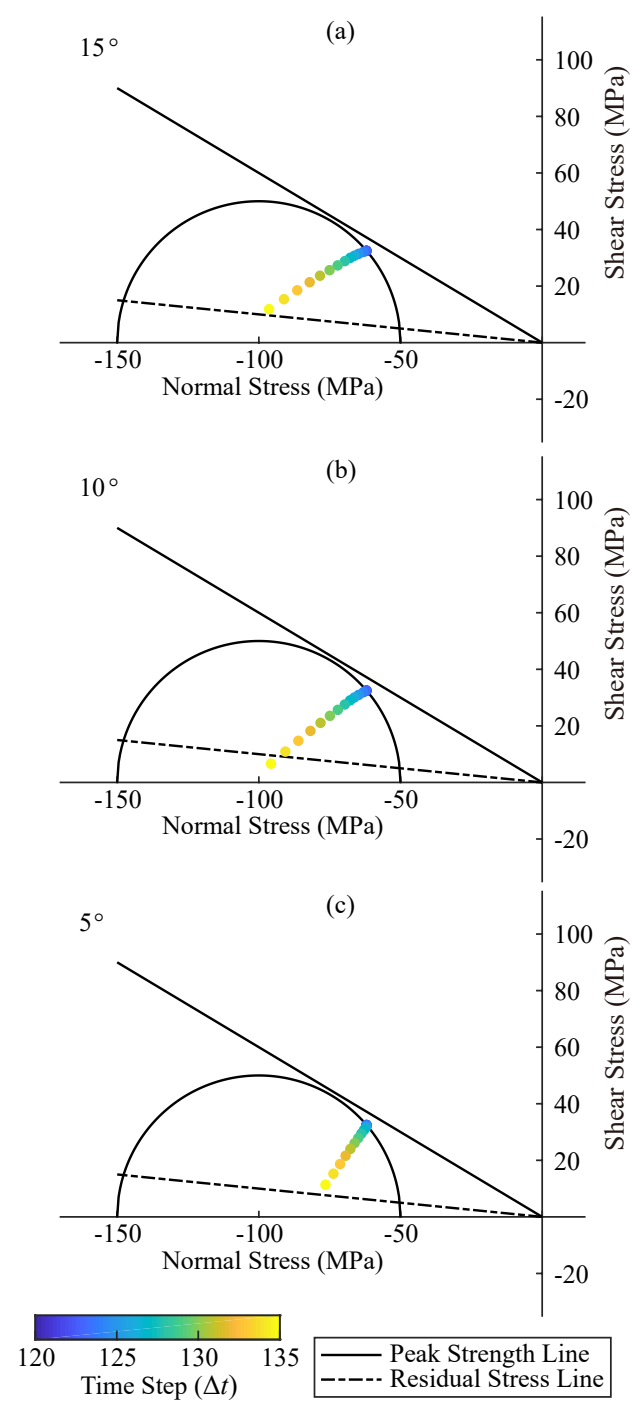

Figure 9. Normal and shear stress on element III at time steps 110 to 135 if we consider only the influence of the rupture of plane $b$. (a) $\left(a_{1}, a_{2}\right)=\left(15^{\circ},-25^{\circ}\right)$, (b) $\left(a_{1}, a_{2}\right)=\left(10^{\circ},-25^{\circ}\right)$, (c) $\left(a_{1}, a_{2}\right)=\left(5^{\circ},-25^{\circ}\right)$. The ordinate and abscissa of a dot represent the shear stress and normal stress on element III, respectively, and the color represents the time of the dot. The rupture on plane $a$ is assumed to propagate to plane $b$ unimpeded.

III moves away from the peak strength line no matter whether the value of $\Delta a$ is large or small, and the medium value of $\Delta a$ causes the stress on element III away from the peak strength line to be the fastest. When $a_{1}=-15^{\circ}$ (Figure $8 \mathrm{f}$ ), plane $b$ cracks very early and begins to produce a negative effect on the rupture of plane $c$, making it difficult for plane $c$ to crack, which is in accordance with the fact that plane $c$ hardly cracks when $\left(a_{1}, a_{2}\right)=\left(-15^{\circ},-25^{\circ}\right)$.

\section{Conclusions}

In this paper, we applied the BIEM to 3-D branching fault systems to simulate the dynamic rupture process on different fault models with different branch angles in two series. Under a given uniform initial stress state, the direction of a plane determines the normal and shear stress on that plane, which affects its tendency
Table 3. Summary of the interactions between the two branch planes.

\begin{tabular}{|c|c|c|c|c|}
\hline Interaction & $\Delta a$ & $\begin{array}{c}\text { Normal } \\
\text { stress }\end{array}$ & $\begin{array}{l}\text { Shear } \\
\text { stress }\end{array}$ & $\begin{array}{l}\text { Suppression } \\
\text { of the } \\
\text { rupture }\end{array}$ \\
\hline \multirow{2}{*}{$\begin{array}{l}\text { Effect of a rupture } \\
\text { on the lower } \\
\text { branch plane on the } \\
\text { upper one }\end{array}$} & Small & $\begin{array}{l}\text { Decreases } \\
\text { slowly }\end{array}$ & $\begin{array}{c}\text { Decreases } \\
\text { quickly }\end{array}$ & Strong \\
\hline & Large & $\begin{array}{c}\text { Decreases } \\
\text { quickly }\end{array}$ & $\begin{array}{c}\text { Decreases } \\
\text { slowly }\end{array}$ & Weak \\
\hline \multirow{2}{*}{$\begin{array}{l}\text { Effect of a rupture } \\
\text { on the upper } \\
\text { branch plane on the } \\
\text { lower one }\end{array}$} & Small & $\begin{array}{c}\text { Increases } \\
\text { quickly }\end{array}$ & $\begin{array}{c}\text { Decreases } \\
\text { slowly }\end{array}$ & Strong \\
\hline & Large & $\begin{array}{c}\text { Increases } \\
\text { slowly }\end{array}$ & $\begin{array}{c}\text { Decreases } \\
\text { quickly }\end{array}$ & Strong \\
\hline
\end{tabular}

to crack. We calculated the direction of the optimal plane and used the Mohr-Coulomb diagram to illustrate that a plane cracks easily if it is close to the optimal plane on the Mohr-Coulomb diagram and is difficult to crack if it is far from the optimal plane.

In a branching fault system with two branch planes, the stress change produced as one of the branch planes ruptures will promote or suppress the rupture on the other branch plane. We investigated this effect by separating the stress change on a branch fault produced by the other branch plane from the total stress change, as summarized in Table 3. After the lower branch plane cracks, it causes both the normal and shear stress on the upper branch plane to decrease. When the angle between the two branch planes is small, it decreases the shear stress rapidly and decreases the normal stress slowly on the upper branch plane, which suppresses the rupture on the plane. In contrast, when the angle becomes larger, the shear stress decreases more slowly and the normal stress decreases more rapidly; thus, the suppression of the rupture on the upper branch plane is weakened. The rupture on the upper branch plane causes the normal stress to increase and the shear stress to decrease on the lower branch plane. The normal stress increases more slowly and the shear stress decreases more rapidly when the angle between the two branch planes becomes larger. Regardless of whether the angle between the two branch planes is large or small, the rupture on the lower branch plane is suppressed by the rupture on the upper branch plane.

\section{Acknowledgments}

This study is supported in part by the National Natural Science Foundation of China (grant no. 41674050) and by the High-Performance Computing Platform of Peking University.

\section{References}

Ando, R., and Kaneko, Y. (2018). Dynamic rupture simulation reproduces spontaneous multifault rupture and arrest during the $2016 M_{\mathrm{w}} 7.9$ Kaikoura earthquake. Geophys. Res. Lett., 45(23), 12875-12883. https://doi.org/10.1029/2018GL080550

Aochi, H., Fukuyama, E., and Matsu'ura, M. (2000a). Spontaneous rupture propagation on a non-planar fault in 3-D elastic medium. Pure Appl. Geophys., 157(11-12), 2003-2027. https://doi.org/10.1007/PL00001072

Aochi, H., Fukuyama, E., and Matsu'ura, M. (2000b). Selectivity of spontaneous rupture propagation on a branched fault. Geophys. Res. Lett., 27(22), 3635-3638. https://doi.org/10.1029/2000GL011560 
Aochi, H., Madariaga, R., and Fukuyama, E. (2002). Effect of normal stress during rupture propagation along nonplanar faults. J. Geophys. Res.: Solid Earth, 107(B2), ESE-5-1-ESE 5-10. https://doi.org/10.1029/2001JB000500

Bhat, H. S., Olives, M., Dmowska, R., and Rice, J. R. (2007). Role of fault branches in earthquake rupture dynamics. J. Geophys. Res.: Solid Earth, 112(B11), B11309. https://doi.org/10.1029/2007JB005027

DeDontney, N., Rice, J. R., and Dmowska, R. (2012). Finite element modeling of branched ruptures including off-fault plasticity. Bull. Seismol. Soc. Am., 102(2), 541-562. https://doi.org/10.1785/0120110134

Dong, S., and Zhang, H. M. (2019). Effects of angles on dynamic ruptures on Ytype branched faults. Chinese J. Geophys. (in Chinese), 62(11), 4156-4169. https://doi.org/10.6038/cjg2019M0572

Eberhart-Phillips, D., Haeussler, P. J., Freymueller, J. T., Frankel, A. D., Rubin, C. M., Craw, P., Ratchkovski, N. A., Anderson, G., Carver, G. A.,... Wallace, W. K. (2003). The 2002 Denali fault earthquake, Alaska: A large magnitude, slippartitioned event. Science, 300(5622), 1113-1118. https://doi.org/10.1126/science.1082703

Feng, X., and Zhang, H. M. (2017). Equivalent formulae of stress Green's functions for a constant slip rate on a triangular fault. Earthq. Sci., 30(3), 115-123. https://doi.org/10.1111/j.1365-246X.2006.03868.x

Hao, K. X., Si, H. J., Fujiwara, H., and Ozawa, T. (2009). Coseismic surface-ruptures and crustal deformations of the 2008 Wenchuan earthquake $M_{\mathrm{w}} 7.9$, China. Geophys. Res. Lett., 36(11), L11303. https://doi.org/10.1029/2009GL037971

Ida, Y. (1972). Cohesive force across the tip of a longitudinal-shear crack and Griffith's specific surface energy. J. Geophys. Res., 77(20), 3796-3805. https://doi.org/10.1029/JB077i020p03796

Kame, N., and Yamashita, T. (1999). Simulation of the spontaneous growth of a dynamic crack without constraints on the crack tip path. Geophys. J. Int., 139(2), 345-358. https://doi.org/10.1046/j.1365-246x.1999.00940.x

Kame, N., Rice, J. R., and Dmowska, R. (2003). Effects of prestress state and rupture velocity on dynamic fault branching. J. Geophys. Res.: Solid Earth, 108(B5), 2265. https://doi.org/10.1029/2002JB002189

Niazi, M. (1986). Inferred displacements, velocities and rotations of a long rigid foundation located at El Centro differential array site during the 1979 Imperial Valley, California, earthquake. Earthq. Eng. Struct. Dyn., 14(4), 531-542. https://doi.org/10.1002/eqe.4290140404

Oglesby, D. D., Day, S. M., Li, Y. G., and Vidale, J. E. (2003). The 1999 Hector Mine earthquake: The dynamics of a branched fault system. Bull. Seismol. Soc. Am., 93(6), 2459-2476. https://doi.org/10.1785/0120030026

Poliakov, A. N. B., Dmowska, R., and Rice, J. R. (2002). Dynamic shear rupture interactions with fault bends and off-axis secondary faulting. J. Geophys. Res.: Solid Earth, 107(B11), ESE-6-1-ESE 6-18. https://doi.org/10.1029/2001JB000572

Qian, F., Wu, B. N., Feng, X., and Zhang, H. M. (2019). 3D numerical simulation of dynamic ruptures on complex fault systems by BIEM with unstructured meshes. Chinese J. Geophys. (in Chinese), 62(9), 3421-3431. https://doi.org/10.6038/cjg2019M0642

Sieh, K., Jones, L., Hauksson, E., Hudnut, K., Eberhart-Phillips, D., Heaton, T., Hough, S., Hutton, K., Kanamori, H.,... Zachariasen, J. (1993). Near-field investigations of the Landers earthquake sequence, April to July 1992. Science, 260(5105), 171-176. https://doi.org/10.1126/science.260.5105.171

Tada, T., and Yamashita, T. (1997). Non-hypersingular boundary integral equations for two-dimensional non-planar crack analysis. Geophys. J. Int., 130(2), 269-282. https://doi.org/10.1111/j.1365-246X.1997.tb05647.x

Xu, D. D., Liu, Z. L., Liu, X. M., and Zhuang, Z. (2016). A numerical study on dynamic shear rupture along frictional faults. Int. J. Damage Mech., 25(1), 69-86. https://doi.org/10.1177/1056789515583342

Zhang, H. M., and Chen, X. F. (2006). Dynamic rupture on a planar fault in threedimensional half space - I Theory. Geophys. J. Int., 164(3), 633-652. https://doi.org/10.1111/j.1365-246X.2006.02887.x 